\title{
Spectral Imaging of Portolan Charts
}

\author{
Fenella G. France, ${ }^{\mathrm{a}}$ Meghan A. Wilson, ${ }^{\mathrm{a}}$ and Anita Ghez ${ }^{\mathrm{a}}$ \\ ${ }^{a}$ Preservation Research and Testing Division, Library of Congress, Washington, District of Columbia; United States of America; \\ frfr@loc.gov,mhill@loc.gov
}

\begin{abstract}
Spectral imaging of Portolan Charts, early nautical charts, provided extensive new information about their construction and creation. The origins of the portolan chart style have been a continual source of perplexity to numerous generations of cartographic historians. The spectral imaging system utilized incorporates a 50 megapixel mono-chrome camera with light emitting diode (LED) illumination panels that cover the range from $365 \mathrm{~nm}$ to $1050 \mathrm{~nm}$ to capture visible and non-visible information. There is little known about how portolan charts evolved, and what influenced their creation. These early nautical charts began as working navigational tools of medieval mariners, initially made in the 1300s in Italy, Portugal and Spain; however the origin and development of the portolan chart remained shrouded in mystery. Questions about these early navigational charts included whether colorants were commensurate with the time period and geographical location, and if different, did that give insight into trade routes, or possible later additions to the charts? For example; spectral data showed the red pigment on both the 1320 portolan chart and the 1565 Galapagos Islands matched vermillion, an opaque red pigment used since antiquity. The construction of these charts was also of great interest. Spectral imaging with a range of illumination modes revealed the presence of a "hidden circle" often referred to in relation to their construction. This paper will present in-depth analysis of how spectral imaging of the Portolans revealed similarities and differences, new hidden information and shed new light on construction and composition.
\end{abstract}

Keywords: Portolan charts, nautical maps, spectral imaging, cultural heritage

\section{Introduction}

The expanded utilization of spectral imaging for noninvasive assessment of cultural heritage has led to applications that enable non-invasive chemical identification of historic materials, the recovery of obscured and degraded non-visible information, and the capacity to monitor non-visible changes in fragile materials to optimize conditions for storage and display. Spectral imaging analysis advances the capability for non-contact chemical characterization of colorants, inks, and substrates through their specific spectral response, aiding provenance of historic artifacts (France and Toth 2011). Monitoring deterioration or changes due to exhibit and other environmental conditions is critical for the longevity of cultural heritage objects. Analysis of spectral images has the added benefit of providing a spectral map locating each individual compound over the entire surface of the object, as opposed to the more common singlepoint analysis of many other chemical analytical techniques (France 2011). The spectral map enables ease of identification and separation of similar colored pigments, and can also detail overlays of various materials.

Spectral imaging was used to assess a series of five Portolan Charts, early nautical charts from 1320-1633 that were used in the Mediterranean for 200-400 years. These charts included: Mediterranean (1320), Europe and the Mediterranean (1484), Mediterranean and Western Europe (Prunes) (1559), Central and South America, Pacific Coast (1565) and the Mediterranean and Western Europe (1633).
The imaging provided extensive new information about the construction and creation of these parchment maps. The origins of the portolan chart style have been a continual source of perplexity to numerous generations of carto-graphic historians. Imaging tools developed for astronomical, remote sensing and medical imaging have been adapted and customized for the recovery of lost and obscured information that can be made visible on degraded cartographic manuscripts. The Library of Congress Preservation Research and Testing Division (PRTD) has developed this application to the preservation and analysis of cartographic materials as a powerful, noninvasive technique to provide access to enhance nonvisible information in layers of registered high resolution digital images, characterize historic pigments and detect change.

There is little information to indicate how portolan charts evolved, and what influenced their creation, and it is interesting that these nautical charts demonstrate a high level of accuracy, somewhat unexpected for their arrival in the latter half of the thirteenth-century. The surviving charts represent the earliest constructed, and studies give clear examples that the Mediterranean's incorporated the use of local knowledge into these charts, and possibly, the improvement of these charts through direct use as trading horizons expanded. In the history of cartography, the invention of the portolan chart was a major revolutionary moment in the history of mapping. These early nautical charts began as working navigational tools of medieval mariners, initially made in the 1300 s in Italy, Portugal and Spain; however the origin and development of the portolan chart remained shrouded in mystery. The style 
used to generate these charts was focused on the facilitation of navigational aids, and incorporated compass directions that created consistent arrays of grids across the entire chart. Research of a number of portolan charts covering the period 1320 to 1633 and these illustrate the overall focus of the nautical charts on the Mediterranean Sea, with one chart, possibly the first of the New World, outlining the pacific coast of Central and South America. Examinations of these Portolan charts confirmed their provenance through radiocarbon dating. One nautical chart of the Mediterranean and Black Seas, believed to be the earliest-known portolan chart of the Western Hemisphere, was prepared by a Genoese mapmaker between 1320 and 1350, while the New World chart vellum dated to ca. 1565 and shows the region from Guatemala to northern Peru, and may be the first chart to represent the Galapagos Islands. Little was known about the pigments and colorants used to create the charts. Spectral imaging can differentiate between pigments that appear visually identical in color and confirm the spectral response without the need to sample. Questions included whether colorants were commensurate with the time period and geographical location, and if different, did that give insight into trade routes, or possible later additions to the charts? For example; spectral data showed the red pigment on both the 1320 portolan chart and the 1565 Galapagos Islands matched vermillion, an opaque red pigment used since antiquity. The construction of these charts was also of great interest. Spectral imaging with a range of illumination modes revealed the presence of a "hidden circle" often referred to in relation to their construction. Mapping and layering of the chart rhumb lines indicated many went over the coastlines and toponyms, always assumed to have been added later in the construction. This paper will present in-depth analysis of how spectral imaging of the Portolans revealed similarities and differences, new hidden information and shed new light on construction and composition.

\section{Spectral Imaging System}

The Library of Congress (LC) conducted spectral imaging with a customized spectral imaging system, a 39 and 50 mega-pixel Monochrome E6 Camera with Kodak CCD sensor $(7216 \times 5412$ pixel array with linear dimension of 6.8 microns), and integrated LED illumination panels containing 16-23 wavebands from the ultraviolet (UV), visible (VIS) and near infrared (NIR) spectral regions $(365 \mathrm{~nm}-1050 \mathrm{~nm})$ developed to image a wide range of collection items. Remote sensing imaging spectroscopy has demonstrated that many features have absorption features $20-40 \mathrm{~nm}$ wide at full width half maximum (FWHM), enabling the spectral imaging system to acquire data for the direct identification of a wide gamut of organic and inorganic materials (Ustin et al. 2004). The full widths at half-maximum (FWHM) of the spectral curves for individual LEDs, range from $6 \mathrm{~nm}$ to $30 \mathrm{~nm}$. Remote sensing imaging spectroscopy has demonstrated that many surface features have diagnostic absorption features that are 20 to $40 \mathrm{~nm}$ wide at FWHM, enabling these spectral imaging systems to acquire data in bands of a width that provides sufficient resolution for the direct identification of a wide gamut of organic and inorganic materials (Goetz et al. 1985). Capturing UV, VIS, and NIR spectral data in reflected, transmitted, and side-lighting illumination modes and orientations allows greater capacity for multivariate image analysis and postacquisition processing. Filters for fluorescence and polarization have been integrated into the system capabilities to expand the current imaging capabilities (Christens-Barry et al. 2001). The image capture generates a cube of fully registered images that can be combined in various combinations to reveal obscured and hidden text, characterize pigments, and with different illumination modes, provide information about specific construction and printing techniques (Emery et al. 2009).

\section{Spectral Imaging Data}

Examinations of one of the earliest known Portolan charts confirmed its provenance through radiocarbon da-ting. Other analyses confirmed the age of the vellum used to create later charts to be commensurate with previously documented time periods from 1484-1633. In the history of cartography, the invention of the portolan chart was a major revolutionary moment in the history of mapping. These early nautical charts began as working navigational tools of medieval mariners and later developed into very stylized and decorative art form (Campbell 1986). Portolan charts were initially made in the 1300s in Italy, Portugal and Spain, however the origin and development of the portolan chart remained shrouded in mystery. PRTD research into five representative portolan charts covering the period 1320 to 1633 illustrate the overall focus of the nautical charts on the Mediterranean Sea, with one chart, possible the first of the New World, outlining the pacific coast of Central and South America. The style used to generate these charts was focused on the facilitation of navigational aids, and incorporated compass directions that created consistent arrays of grids across the entire chart. The nautical chart of the Mediterranean and Black Seas, believed to be the earliestknown portolan chart of the Western Hemisphere, was prepared by a Genoese mapmaker between 1320 and 1350, while the New World chart vellum dated to ca. 1565. This sixteenth-century sailing chart of the Pacific Coast of Central and South America shows the region from Guatemala to northern Peru, and may be the first chart to represent the Galapagos Islands, off the coast of present-day Ecuador.

Little was known about pigments and colorants used to create the charts. Spectral imaging was utilized to assess and characterize components of these charts to begin to address a range of questions. Were colorants commensurate with the time period and geographical location? If differences from what was expected were observed, did these give insight into trade routes, or possible later additions to the charts? Characterization of colorants present on the chart was confirmed through the use of additional non-invasive analyses, including portable x-ray fluorescence and x-ray diffraction. This minimized handling of the artifact and additional identification 
enabled confirmation of the accuracy of spectral imaging reference materials, as well as suggesting a source or location for the colorants (Kelley 1999).

\subsection{Pigment and Colorant Analyses}

Spectral imaging can be utilized to differentiate between pigments that may appear visually identical in color. The spectral imaging system can confirm the spectral response without the need to sample, whereas if only one pigment location were sampled, it may overlook that though visually similar, the compounds are chemically differ-ent. This can lead to misidentification of materials present within the object, as well as the potential to overlook different materials if they appear visually similar. This capacity to assess the spatial distribution of materials augments the knowledge of the object allowing layers of information to be mapped.

As shown in Figure 1, more information can be made visible through this processing technique. The upper image shows what can be seen with the unaided eye, the lower image illustrates the same section of a map after processing where the faint green lines now appear broader (purple in the pseudocolor processed image) and indicate the spread of verdigris - a corrosive copper containing pigment - into the parchment causing potential damage. Other multi-variate image processing techniques can be used to enhance obscured text, or heavily redacted texts.

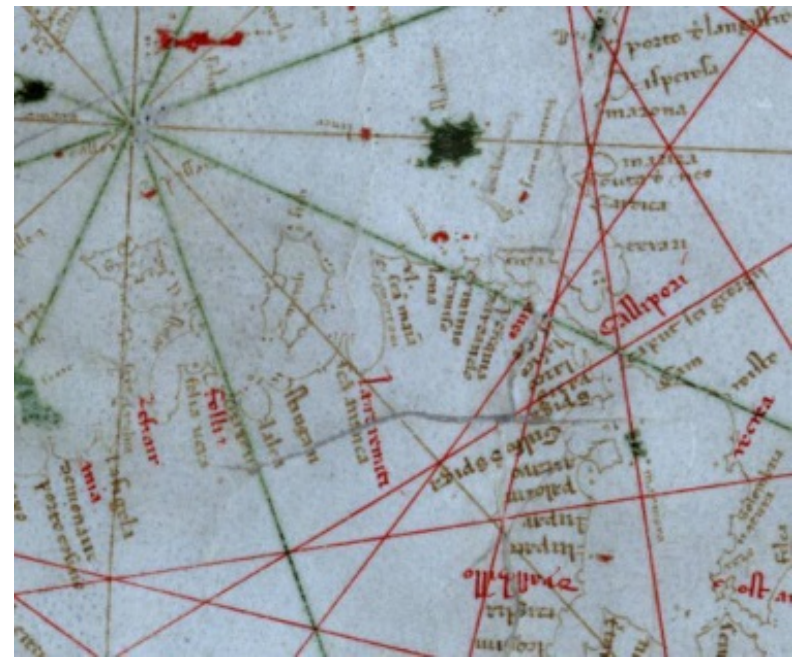

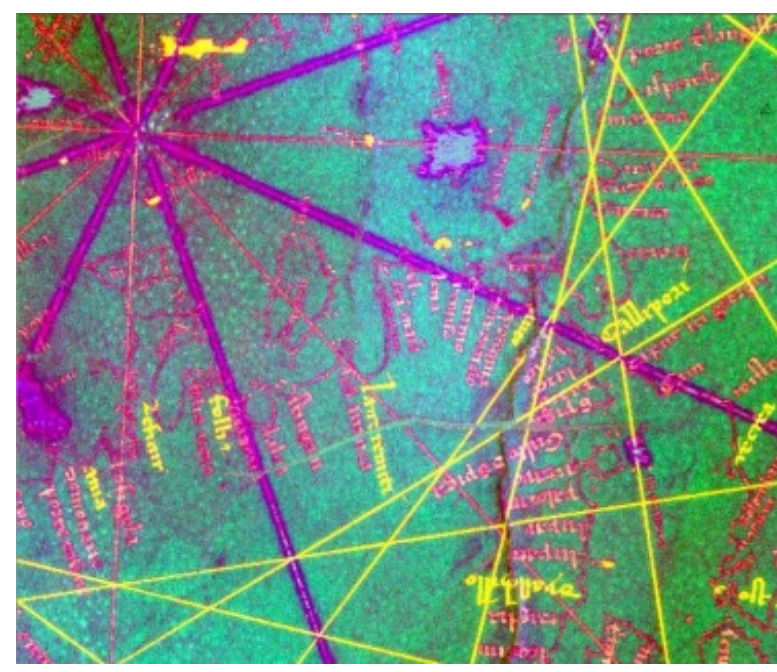

Figure 1. Principal Component Image Processing: Full Spectrum Color Image (Left) False Color Image (Right)

Spectral data for comparative assessment of the colorants present on the Portolan charts were obtained from a range of naturally aged pigment reference samples. The figure below illustrates results obtained for a selection of red pigments compared to the spectral response obtained from a region of a red line on the 1320 portolan chart (data shown is an average sample of a range of data points selected). Spectral data matched well for vermillion, an opaque red pigment used since antiquity, originally derived from the powdered mineral cinnabar. The synthesis of vermillion from mercury and sulfur may have been invented by the Chinese, with the synthetically produced pigment used throughout Europe from the 12th century. The technique for synthesizing vermillion became widely known in Europe by the 14th century, greatly reducing the cost (Zeld 1993).

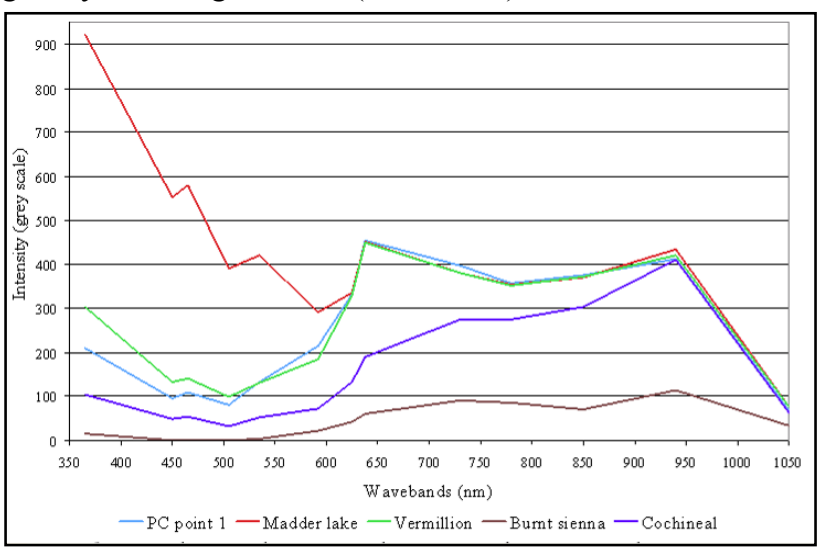

Figure 2. 1320 Portolan Chart Spectral Comparison Graph

Since the 1320 chart was on exhibition when additional analyses were undertaken, data for red pigments comparisons were undertaken for the 1565 South American portolan chart. These fragile manuscripts could not be sampled for pigments, and analysis of spectral, Xray fluorescence and X-ray diffraction data allowed researchers to characterize pigments and determine provenance of the artifacts. When compared with x-ray diffraction, there was a clear positive for cinnabar on the red of the Galapagos Islands, while x-ray fluorescence 
confirmed the presence of mercury in the non-invasive testing. These analyses further confirmed the results obtained with the hyper-spectral imaging utilizing the comparison with spectral reference samples. While these charts were usually created in Europe and used to depict travel in the Mediterranean, the 1565 Portolan was a very early chart probably created in South America. This gave credence to the hypothesis that this document had been created in the America's rather than in the Mediterranean. Further analysis of the 1565 Portolan chart revealed the use of a local indigenous pigment (indigo) on the 1565 chart, later over-painted with another blue pigment azurite, data only able to be separated through spectral mapping of the entire map.

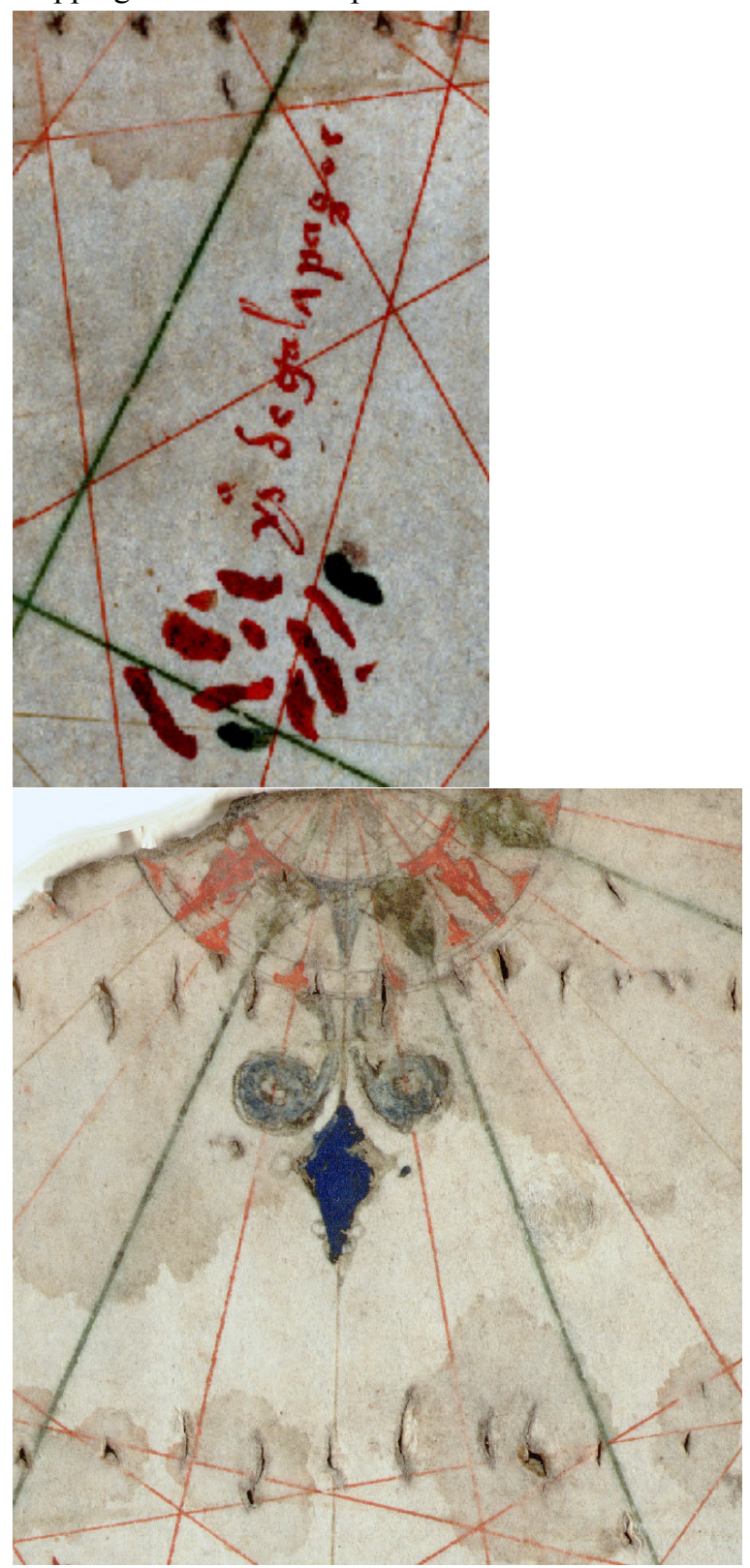

Figure 3. 1565 Portolan Chart: Galapagos Islands (Left), Indigo and Azurite Blue Pigments (Right)

\subsection{Other Spectral Features}

Spectral imaging can be used to capture a wide range of information, and the high resolution images often allow new data to be revealed that was previously unknown. One of the questions about these portolan charts was how they were constructed, what obscured text or data could be revealed, and how were they used. Raking or sidelighting in the visible and infrared regions greatly assists the capture of construction markings, while processing can also capture how layers of information were laid down on the charts.

It has been proposed that the analysis of visual and technological style can greatly impact the choices made by artists and artisans, with the selection of materials and specific techniques of construction being related to appearance and function (Ash 1982). This framework aids the integration of visual and technological components, a critical aspect for the Waldseemüller 1507 World Map and Portolan charts that bridges the gap between function and display. The Waldseemüller still depicts aspects that reflect the earlier mappamundi style, while Portolan charts reflect a turning point in the history of cartography (Woodward 1990). The late medieval mappamundi reflect theological aspects and tend to display a view of the world that was somewhat dominated by these concerns. In relation to technique, the portolan charts capture and illustrate the Mediterranean seamen's intimate knowledge of the water, the coastlines, ports and areas of danger (vigia) (Pruett 1996). The charts were analyzed to assess how these markings had changed over the centuries. Data tended to suggest that once a vigia was introduced to the charts it tended to stay there, although as sand bars and rocks shifted, the area may be shown as having reduced or enlarged in area. The charts were analyzed to track how these markings changed or remained the same through the 300 plus years under study.

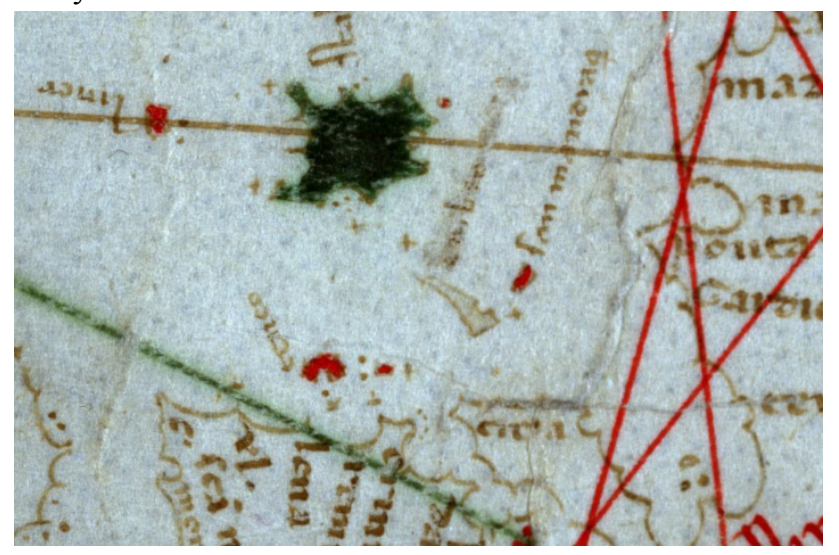

Figure 4. Crosses in Center of Image Indicating "Vigia" or "Danger"

Since the charts were of practical importance and used daily, the few that remain often reflect the other aspect of the Portolan chart - a map created for a sponsor or person of note to have as a decorative wall-hanging. The presence of gold on a number of the charts, and the absence of any salts indicating working charts used on board ships, tends to support this premise. This represents a dichotomy - from one perspective a critical working tool, from another, the morphing into an object of desire 
by sponsors or persons of wealth. These and other Portolan charts continue to be examined in collaboration with cartographers, historians and curators. The investigations include the assessment of regional variations in pigment use, changes and similarities of materials used throughout the 300-year period under examination, and the depiction of figures and other stylistic features as repeated and changing representational images on the Portolans charts. This was the first time spectral imaging and image analysis was used to attempt to fully understand the interactions between map-makers and their location, including their choice of materials and the methods of construction as they relate to cultural and geographical differences. If the charts had been used on board ship, evidence of salt from spray would be expected, unless they had been treated significantly.

The scientific study of place names (toponyms) analyzes their origins, use and meanings. This is a critical component for determining provenance of early maps, since geographical locations, their spelling and changes in place names, was an important method for assessing the creation date of a map. Scholars have found that toponyms provide valuable insight into the historical geography of a particular region. The analysis of toponym development in the production of pre-1450 charts illustrates a relatively standardized core region, while recording the creation and abandonment of coastal settlements. Finding and utilizing place-names during analysis of the charts helped determine the stability of these names relative to recorded areas of danger on coastal areas. In some areas, degraded text could be restored with digital enhancement and recovery by image processing, aiding scholars in translating and tracking these changes. Multivariate image analysis of deteriorated provenance data from the toponyms (place-names) allowed confirmation of accurate dating for the 1320 chart.

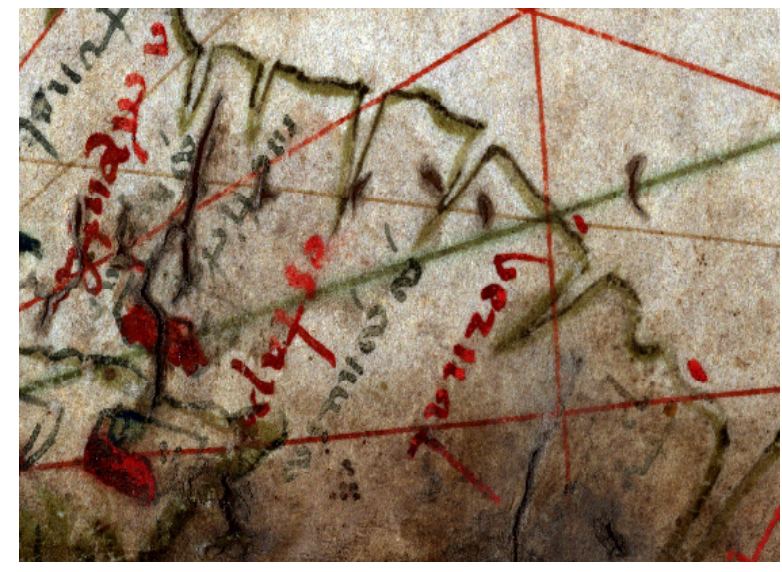

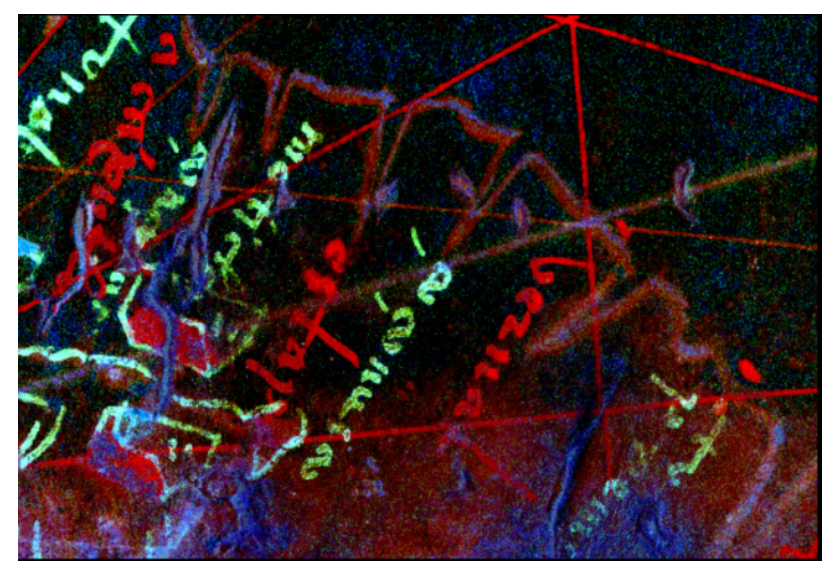

Figure 5: Full Spectrum Color (Left) and Principal Component Analysis (PCA) Pseudocolor Image of Toponyms (Right)

Although there are distinctions between the sciences and the humanities, the critical overlap and multidisciplinary approach required to analyze cultural heritage is often overlooked. While the social sciences focus their concerns on civilization patterns, cultural traditions and political and moral interests, these factors are all influenced by the specific culture and era in which they exist (Karnaukhov 2010). Detaching the understanding of how objects were created during historic periods creates a separation of contiguous knowledge, a gap that scientific analyses aptly bridge, adding to the knowledge and understanding of the object. Historically it was considered that to generate the portolan chart, the rhumb lines would be laid down first, then the coastlines, followed by the coastal place names. As illustrated in figure 6 , the processing indicates that in some instances the lines appear to go over the text and coastlines, indicating a different layering of the information. This led to the discussion of the reference in some instances to the hidden circle or the use of a template for the construction of these charts.

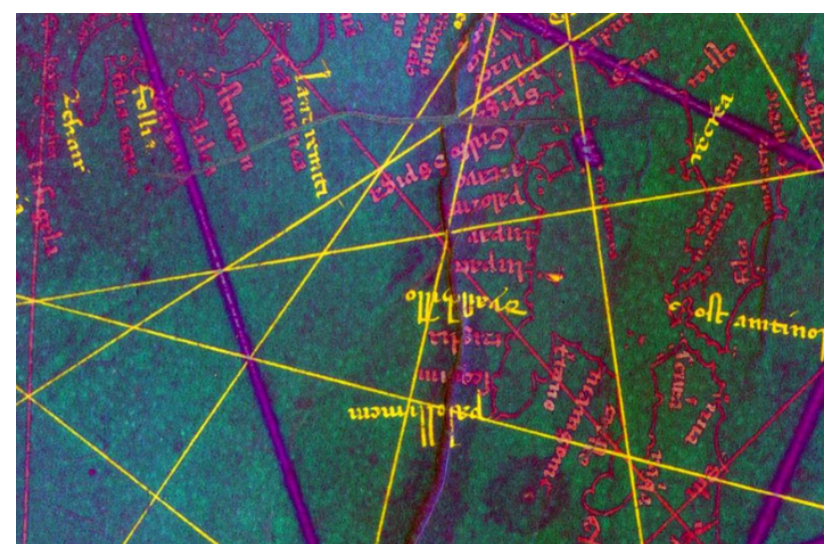

Figure 6: Principal Component Analysis (PCA) Pseudocolor Image Illustrating Layers of Information on Portolan Chart

A precise study of the exact location of any compass piercings of the parchment was made and it was found that when connected, on one chart, these piercings were all located on the circumference of a circle. In addition, the circle extended past the actual size of the parchment itself, lending support to the theory of a template. To further support this theory, the accuracy of the distances 
throughout the same regions of the Mediterranean over a period of 200-300 years was very similar, further corroborating the template theory (figure 7).
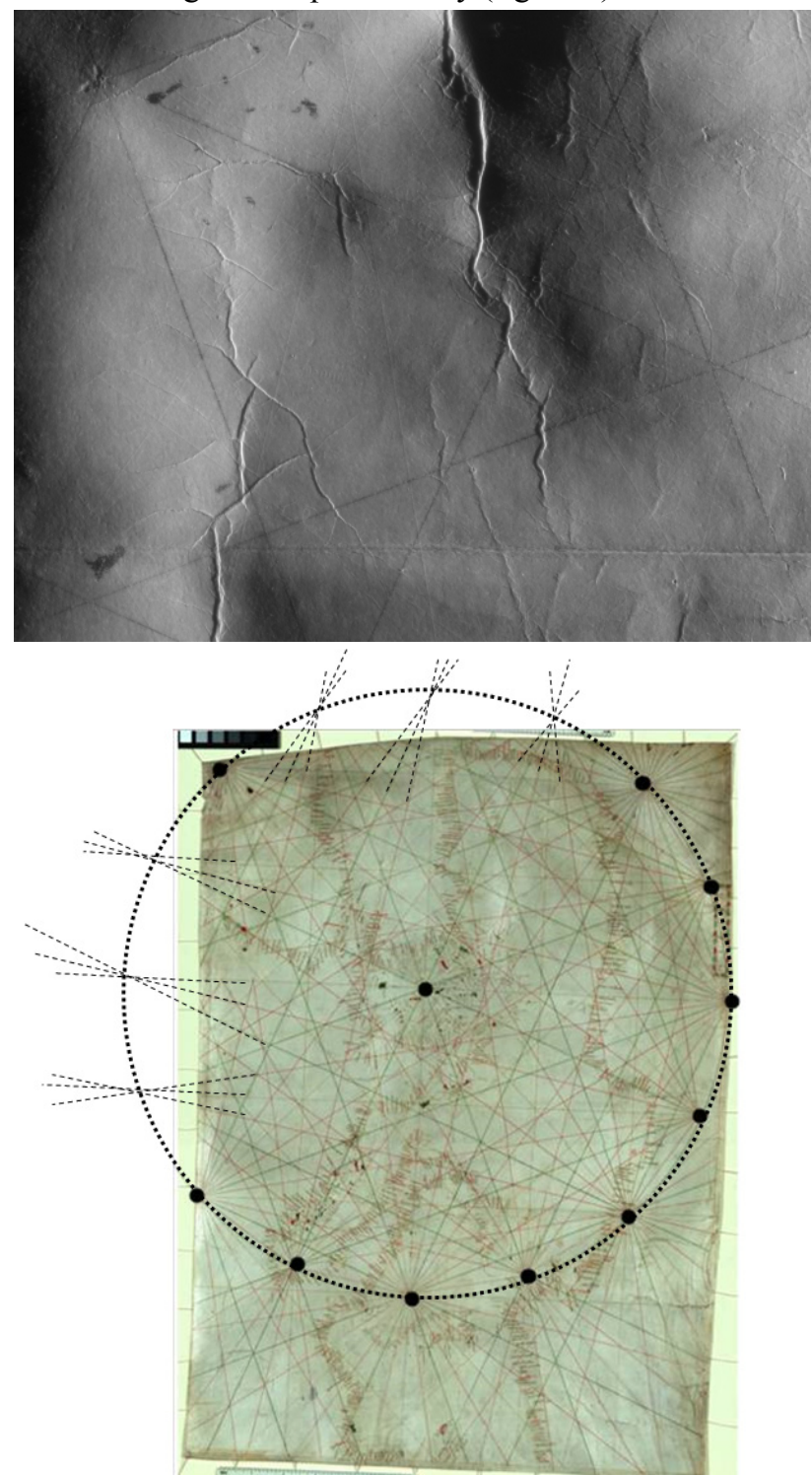

Figure 7: Infrared Side-lighting Image (Left) and the "Hidden Circle" (Right)

\section{Conclusions}

Multivariate image analysis of spectral assessment of cultural heritage objects with principal component analysis, spectral curves and other image processing techniques provides a powerful tool for non-invasive monitoring and investigative research of documents and objects in storage, on exhibit, and after or during treatments. This spectral data allows the mapping of spectral responses across the surface of heritage materials, allowing the linking of spectral data with a range of complementary point-source analytical techniques. The linking of standardized data sets enables researchers to interpret results from a broader range of data, ensuring a multidisciplinary and collaborative approach to the preservation of cultural heritage objects. The capacity to assess the spatial distribution of materials greatly augments an objective understanding of the heritage material, allowing layers of scientific, cultural, techno-logical, and historical information to be integrated and mapped. Image analysis of spectral data serves as an effective examination and analysis tool for the noninvasive characterization of substrates and colorants in a range of heritage material types - from paper and parchment to ceramics. A standardized approach to image acquisition procedures and processing parameters has taken spectral imaging from sophisticated remote sensing to an easily accessible laboratory tool for the preservation of heritage materials.

The transfer of technologies from multidisciplinary fields has demonstrated huge potential for the cultural heritage arena, particularly in the expansion of non-invasive analytical techniques. This knowledge transfer reiterates the utilization of non-invasive modalities for addressing the preservation of original materials - both substrate and media. Expanding the original use of the technology to encompass and answer additional preservation and scholarly questions ensures greater use of resources, and enables preservation scientists to fully interrogate instrumental techniques with expanded applications. Spectral imaging therefore has a two-pronged approach for heritage preservation - information to preserve the original, and recovering and acquiring baseline information to monitor and prevent further irreversible deterioration occurring. Multivariate image analysis techniques are critical for capturing non-visible data that can potentially advance preservation of cultural heritage, with the linking of spectral and related analyses expanding the scope of analysis of heritage materials. Expanding our knowledge of historic cartographic materials greatly advances our knowledge of how these maps were used in a historical context and the importance of these materials to the history of cartography, both from a practical perspective, and how they were respected and utilized throughout previous centuries.

\section{Acknowledgements}

We are grateful to PRTD staff and interns for additional assistance with analyses.

\section{References}

Ash, N.E. Recording Watermarks By Beta-Radiography and Other Means. The Book and Paper Group, The American Institute for Conservation, Volume (1982) http://cool.conservation-

us.org/coolaic/sg/bpg/annual/v01/bp01-02.html

Campbell, T. Census of pre-sixteenth century portolan charts. (1986). Imago Mundi, 38, 67-94.

Christens-Barry, W.A, Boydston, K, and Easton, Jr. R.L. (2010), Standardized System for Multispectral Imaging Proceedings of SPIE-International Society of Optical Engineering 7528, 0M.

Emery, D., France, F.G. and M.B. Toth, (2009) Management of Spectral Imaging Archives for Scientific Preservation Studies, Proceedings of Imaging Science and Technology (IS\&T): Archiving 2009, $137-$ 141. 
France, F.G, (2011). Advanced Spectral Imaging for Non-invasive Microanalysis of Cultural Heritage Materials: Review of Application to Documents in the U.S. Library of Congress, Appl. Spectroscopy., 65(6), 565-57.

France, F.G. and Toth, M.B. (2011). Spectral Imaging for Revealing and Preserving World Cultural Heritage, Proceeding of 19th European Signal Processing Conference, EUSIPCO, 1450-1454.

Goetz, F.H., Vance, G., Solomon, J.E, and Rock, B.N. (1985). Image Spectroscopy for Earth Remote Sensing, Science (Washington, D.C.) 228, 1147-1153.

Hawkins, D.A. (2009). Review: Masonic Symbols In The L'Enfant Plan, Washington History, 21, 100-105.

Kelley, J.E. (1999). Curious Vigia in Portolan Charts, Cartographica, Vol 36(1) 41-49.

Pruett, L.P. (1996). Sixteenth-Century Manuscripts in Brussels, Berlin and Vienna: Physical Evidence as a Tool for Historic Reconstruction, Revue belge du Musicologie / Belgisch Tidjschrift voor Muziekwetenschap, 50, 73-92.

Ustin, S.L, Roberts, D.A., Gamon, J.A, Asner, G.P. and Green, R.O. (2004). Using Imaging Spectroscopy to Study Ecosystem Processes and Properties, Bioscience, 54(6), 523-534.

Verner, C. Surveying and mapping the new Federal City: The first printed maps of Washington, D.C. (1969). Imago Mundi, 23, 59-72.

Woodward, D. The correlation of watermark and paper chemistry in sixteenth century Italian printed maps (1990). Imago Mundi, 42, 84-93.

Zald, M.N. (1993). Organization Studies as a Scientific and Humanistic Enterprise: Toward a Reconceptualization of the Foundations of the Field, Organizational Science, 4(4) 513-528.

Karnaukhov, V. (2010). Methods and Tools for Watermark Digital Processing, Eikonopoiia: Symposium on Digital Imaging of Ancient Textual Heritage, Helsinki, Finland, 115-128. 